Title: Validity of wrist worn accelerometers and comparability between hip and wrist placement sites in estimating physical activity behaviour in preschool children.

\title{
Authors:
}

Jane Hislop ${ }^{1}$, Nicole Palmer ${ }^{2}$, Priya Anand ${ }^{3}$, Tara Aldin ${ }^{4}$

${ }^{1}$ School of Health Sciences, Queen Margaret University, Edinburgh, Scotland, United Kingdom ${ }^{2}$ Physiotherapy Department, Stirling Community Hospital, Stirling, Scotland, United Kingdom

${ }^{3}$ The Injury care clinics limited, Solihull, West Midlands, England, United Kingdom

${ }^{4}$ Physiotherapy Department, Sighthill Health Centre, Edinburgh, Scotland, United Kingdom

\section{Email: jhislop@qmu.ac.uk}

Short title: Validity and comparability of wrist worn accelerometers in preschoolers

Key words: Physical activity, pre-school, Actigraph, validity, accelerometry

\section{Work was carried out at Queen Margaret University Edinburgh.}




\begin{abstract}
Wrist-worn accelerometers can increase compliance with wearing accelerometers, however, several large scale studies continue to use hip- worn accelerometers and it is unclear how comparable data is from the two sites. The study aims were: to investigate agreement between wrist- and hip- worn accelerometers and to determine the validity of Johansson et al cut-points for wrist worn accelerometers in preschool children.

A sample of 32 preschool children (21 boys, 4.2 (0.5) years, BMI 16.6 (1.1)) were videoed wearing GT3X+ accelerometers on their wrist and hip while they engaged in 1 hour of free-play in their nursery. Children's activity were coded using, the Children's Activity Rating Scale (CARS): with CARS, level 1 'sedentary' and levels 2 to 5 were classified as time spent in TPA. Accelerometry data were processed using Johansson et al cut-points for the wrist data and Evenson et al cut-points for the hip data, into time spent in different intensities of PA. The mean counts per minute (cpm) from the hip and wrist were compared.

There was a strong correlation between the hip and wrist cpm $(r=0.81, \mathrm{p}<0.01)$ and total count data $(r=0.83 \mathrm{p}<0.01)$, however there was a large systematic bias with wide limits of agreement. Good agreement (mean difference (LOA) $1.1(-9.9,12.1)$ was found between the CARS estimate of TPA (29.5 (10.4) mins) and the wrist estimate, using the Johansson et al cut points (28.4 (9.8) mins). There was also a reasonable agreement between the hip estimates with the Evenson et al cut-points and Johansson et al estimate (mean difference (LOA):6.3 (-8.8, 21.4) mins.

In conclusion, the findings suggest that the Johansson et al (2013) cut-points applied to wrist worn accelerometers provides a valid estimate of TPA in preschool children and have reasonable agreement with Evenson et al cut-points applied to hip accelerometers.
\end{abstract}




\section{Introduction}

Declining levels of physical activity have been attributed to the dramatic increase in childhood obesity in the UK since the 1980s (Parsons et al 1999, Cox et al 2012) and while levels of obesity in child and adult populations have plateaued in recent years, it is maintained that resolving the childhood obesity 'epidemic' should remain a public health priority (Rokholm et al 2010).

The pre-school years ( 3 to 5 years of age) are argued to be one of the critical periods of childhood during which the long term regulation of energy balance may be programmed (Dietz 2001). This, together with the fact that lifestyle behaviours are thought to track from pre-school to childhood, and subsequently into adulthood (Biddle et al 2010, Malina 1996) means that the early years may be a critical time for promoting physical activity and preventing sedentary habits developing (Goldfield et al 2012).

Current recommendations for health are for pre-school children to undertake at least 180 minutes (3 hours) of daily physical activity which should include both light and energetic activities, such as running, swimming and skipping and that time spent being sedentary should be minimised (Department of Health, Physical Activity, Health Improvement and Protection 2011, Australian Government, Department of Health and Ageing 2010, Canadian Society of Exercise Physiology 2012, National Association for Sport and Physical Education 2009, National Institute for Health and Clinical Excellence 2009). The focus of these guidelines is for young children to achieve a daily amount of physical activity rather than being concerned with the intensity of that physical activity (Department of Health, Physical Activity, Health Improvement and Protection 2011).

The findings of population based studies, which have described levels and patterns of physical activity and sedentary behaviour in young children, are conflicting and have either reported that preschool children are highly sedentary (Hinkley et al 2012, Jackson et al 2003) and are not meeting physical activity recommendations (Hinkley et al 2012, Alhassan et al 2007, Okely et al 2009, Taylor et al 2009) or that they are meeting physical activity recommendations for health (Heelan and Eisenmann 2006, Janz et al 2002, Janz et al 2004, Martinez-Gomez et al 2009, Metallinos-Katsaras et al 2007, Obeid et al 2011, Telford et al 2005).

These inconsistencies between the findings of studies may be an artefact of the different measurement methods adopted and the variety of different methodological decisions used between studies. It is 
argued that there is a need for consensus on the methodological decisions which influence accurate quantification of physical activity in young children (Cliff et al 2009, Ojiambo et al 2011).

Accelerometers offer an objective means of quantifying physical activity and hip worn accelerometers have been validated for use with preschool children (De Decker et al 2013, Adolph et al 2012, Pate et al 2006, Pfeiffer et al 2006, Hislop et al 2012). Accelerometers measure acceleration of the body part to which they are attached (de Vries et al 2009). It is argued that acceleration of the body is directly proportional to the muscular forces generated, which relates directly to energy expenditure (Freedson and Miller 2000) although their ability to accurately estimate energy expenditure in young children is questionable (de Vries et al 2006).

While several models of accelerometer are commercially available, to date the most frequently used accelerometer in studies of children has been Actigraph accelerometers (Actigraph, Fort Walton Beach, FL, USA) (de Vries et al 2009, Pate et al 2010, Trost et al 2011). One of the methodological decisions when using accelerometers is the decision over which position to place the accelerometer during data collection (Cliff et al 2009). Until recently, studies involving pre-school children have positioned Actigraph accelerometer over the hip during data collection. The rationale being that this attachment places the accelerometers close to the centre of body mass and aligns the sensitive axis with the vertical plane (Puyau et al 2002). However, a recognised limitation of hip worn accelerometers is that they are unable to take into account the increased energy expenditure which occurs with upper limb movements (Janz et al 2006) and as a consequence can underestimate activity (Cleland et al 2013). Following a review of the evidence, Cliff et al (2009) recommended the hip position for the placement of accelerometers during data collection. However, the authors acknowledged that evidence for the optimal position of accelerometers is limited and further investigation is required (Cliff et al 2009).

In recent years there has been growing support for positioning accelerometers at this wrist to take account of upper limb movement and in addition, it is argued that the wrist position will help to improve compliance with wearing accelerometers (Routen et al 2012). Good compliance with accelerometry wearing is essential in reducing drop-out of participants from large-scale population surveys so that the results are representative of a population's activity levels (Rowlands et al 2014). As a consequence some large scale population based studies such as the NHANES study have moved to using wrist worn accelerometers as opposed to waist worn accelerometers (Rowlands et al 2014). 
Johansson et al (2013) calibrated wrist worn accelerometers in toddlers aged 2-3 years and proposed 'cut-point' thresholds to classify accelerometer data into time spent in different intensities. The authors concluded that wrist worn accelerometers offer a feasible alternative placement site to the hip. However, what this study did not do was to compare accelerometer data collected at the wrist with accelerometer data collected from the hip and it is unclear if data collected at these two different placement sites are comparable. This is important as several on-going, large scale longitudinal accelerometry studies have been using and continue to use, hip worn accelerometers (Basterfield et al 2011, Mattocks et al 2010). Greater understanding of how accelerometry output compares, between hip and wrist position, is important to be able to make longitudinal comparison of physical activity behaviour using data which has already been collected, for example the International Children's Accelerometry Database, has 44,454 Actigraph data files, from 20 countries of children aged 3-18 years which have been collected using hip worn Actigraph accelerometers (Sherar et al 2011).

While other models of accelerometers have been calibrated for the wrist position in older children (Djafarian et al 2013) there is limited evidence of how the output compares with that of accelerometers worn at the hip. In one study, Routen et al (2012) compared the Actiwatch worn at the wrist against an Actiwatch worn at the hip in 24 children (mean \pm SD:11.2 \pm 0.5 years). The authors found that accelerometer sites were not comparable with the volume of activity (activity counts) being greater at the wrist compared to the hip and with time spent in light, MVPA, and vigorous being higher with the wrist worn accelerometers. Ekblom et al (2012) explored indirect calorimetry as criterion method for energy expenditure in 22 children 8-10 years when comparing Actiwatch (worn on the wrist) against hip worn uniaxial 7164 Actigraph accelerometer and found while there was correlation in 'counts' between accelerometers there was a systematic difference observed such that the output from the accelerometers can not be used interchangeably. Rowlands et al (2014) found strong correlations between the wrist worn GENEActiv for average counts per day in a study of 58 children aged 10-12 years recorded over 7 days for time spent in sedentary behaviour and MVPA against waist worn GT3X+ for both vertical (uniaxial) and vector magnitude (triaxial) output. Finally, while Trost et al (2014), using pattern recognition approach to analysis of accelerometry data, concluded that the output from the wrist worn GT3X+ accelerometer performed as well as a hip worn accelerometer in 52 children (mean age $13.7 \pm$ 3.1 year), other studies have reported inconsistencies in raw acceleration data between the wrist and hip during structured activities (Hildebrand et al 2014). 
In summary, there is recent debate about whether wrist worn accelerometers instead of hip worn monitors may improve compliance (Routen et al 2012) as well as account for energy expenditure from upper limb movements. While the Actiwatch has been available for a number of years, the Actigraph GT3X+ is now available as a wrist worn monitor. Research is therefore needed to calibrate wrist worn accelerometers such as the GT3X+ and to explore the relationship of data from wrist worn accelerometers with data from Actigraph hip worn accelerometers. Studies which seek to understand how accelerometry output compares are important if there is to be any meaningful longitudinal analysis of physical activity and sedentary behaviour of populations over time.

The aims of this study were:

1) To investigate the relationship between wrist- and hip- worn Actigraph accelerometers output preschool children during free-play

2) To investigate the validity of the Johansson et al (2013) cut-points for wrist worn Actigraph accelerometers in preschool children aged 3-5 years.

\section{Method}

Data were collected from a convenience sample of 32 children aged 3 to 5 years recruited from pre-schools in Edinburgh (21 boys, 4.2 (0.5) years, height: 105.3 (6.1) cm, weight: 18.4 (2.6) cm, BMI $\left.16.6(1.1) \mathrm{kg} / \mathrm{m}^{2}\right), 90 \%$ of the sample were classified as 'healthy' weight and $10 \%$ as overweight/obese i.e. BMI at or above $85^{\text {th }}$ centile relative to UK population reference data (Cole 2002).

To recruit participants flyers were distributed to parents at the pre-school inviting their children to take part. Healthy children, aged between 3 to 5 years, were included. Children with any known neurological, respiratory or musculoskeletal problem, which would affect their mobility, were excluded (Hislop et al 2012). Prior to taking part, parental written informed consent and the child's verbal assent to participate was obtained. Ethical approval for the study was granted from the host institution.

Children were video recorded while they engaged in 1 hour of free-play during their usual play-time in the nursery setting. In the nursery, children had access to an outdoor play area where they could play with outdoor toys including carts, scooters and balls. In addition, there was a climbing frame available. In an indoor area children had access to small tables and chairs where they could engage in drawing and crafts. There were books and small toys such as cars and building blocks on the floor, and children also had access to a sand box which was positioned approximately at the child's waist height, so that the child was in a standing position while playing. During the video recording children could freely choose their 
activities and move between these. They were therefore able to engage in sedentary and light activities, in sitting and standing, as well as moderate to vigorous activities such as running, skipping, hopping and climbing. Each child wore two GT3X ${ }^{+}$accelerometers, one on an elasticated belt around their waist positioning the accelerometer over their hip in the mid-axillary line. A second GT3X ${ }^{+}$accelerometer was positioned on the participant's non-dominant wrist. Accelerometers were pre- set to record data in $1 \mathrm{~s}$ epochs which was reintegrated into 5-s epochs.

The Children's Activity Rating Scale (CARS)(Puhl et al 1990) is a direct observation scale which is argued to be the 'gold standard' method of measuring physical activity behaviour in preschool children (Sirard et al 2005). Using the video data children's activity were coded using the CARS method adapted from the original study which used 1-minute sampling period to using a 5-s sampling period (Puyau et al 2002). This approach involved coding each child's activity on a scale of 1 to 5 depending on level of intensity of the activity and then averaging the score over the 5-s period. Similar to the original study each level of coding was only used once during a 5-s coding period. Researchers were trained in using the coding system using video examples and reliability between coders was assessed at the start and end of the data collection period using randomly selected 10 minute extracts of data. The intra-class correlation coefficient was found to be 0.96 .

Table 1 outlines the codes used to classify the observed activities and these were averaged over the 5-second epoch. The intensity of the activity in each epoch was interpreted as outlined in the Johansson et al (2013) study whereby level 1, which included any sitting or reclined activities was classified as sedentary behaviour. Level 2, which included standing activities and level 3, which included slow walking, were grouped together and classified being low or light intensity physical activity. Finally, level 4 which included brisk walking, and level 5, which included running, were classified as high intensity physical activity. Similar to earlier studies with preschool children, activities at levels 4-5 were grouped together and classified as being part of MVPA (Hislop et al, 2012) and levels 2 to 5 were grouped together as part of 'total physical activity' (TPA). 
Table 1: Children's Activity Rating Scale (CARS) 5-point scale to categorize intensity of physical activity.

Level 1: $\quad$ Stationary/motionless

Level 2: $\quad$ Stationary/movement of limbs or trunk (very easy)

Level 3: $\quad$ Translocation (slow/easy)

Level 4: $\quad$ Translocation (medium speed/moderate)

Level 5: $\quad$ Translocation (fast or very fast/hard)

Puhl et al (1990)

The study took place in the preschools' outdoor and indoor play areas and cameras were set up to capture children as they moved between different areas. If a child was out of screen shot the 5-s epoch was excluded from coding and the corresponding accelerometer period was excluded. The cameras were synchronised with the PC set to internet time and the PC was used to initialise the Accelerometers.

The accelerometry count data were processed using the ActiLife software (ActiGraph, Pensacola, FL) applying cut-points to classify activity into time spent in sedentary behaviour, light intensity, moderate to vigorous (MVPA) intensity and total physical activity (TPA). As this study was interested in total physical activity the Johansson et al (2013) cut-points for TPA were applied to the wrist data ( > 221 counts per $5 \mathrm{~s}$ ) and the Evenson et al (2008) cut-points were applied to the hip vertical vector data (Y-Axis) to provide estimates of time spent in TPA (TPA ${ }^{\mathrm{Ev}}>8$ counts per 5 seconds). The Evenson $e t$ al (2008) are frequently adopted in studies of preschool children and have been calibrated in children aged 5 - 9 years. The total counts from the hip vertical vector output and the vector magnitude (VM) output as well as the wrist vector magnitude output was collected.

Data were imported into SPSS (version 21) for analysis. Normality tests were conducted using the Shapiro-Wilks statistic as the sample was less than 50. All data was found to be normally distributed $(p>0.05)$.

Correlational analysis was undertaken to explore the association between the output from the hip and the wrist vector magnitude.

To assess the accuracy of the different accelerometry cut-points as an absolute measure of physical activity, comparison was made between the number of minutes of sedentary behaviour, light intensity, 
MVPA intensity and TPA as estimated by the different cut-points, applied to accelerometers worn at the wrist and hip and then compared with the estimates as determined by the CARS criterion measure of direct observation. The Bland and Altman approach was used to examine the relationship between the minutes estimated using the cut-points and the estimates using the criterion measure (Dale et al 2000).

\section{Results}

An average of 36 (11.2) minutes of accelerometer data was recorded and from this a total of 13681 , 5-second epochs were coded using the CARS. The mean VM cpm recorded at the wrist were 7222.1 (2224.8) cpm and at the hip were $2245.7(852.3) \mathrm{cpm}$. The mean total counts from the accelerometers are presented in table 2 .

Table 2: Mean total accelerometry counts

\begin{tabular}{lcc}
\hline & Mean total counts & (SD) \\
\hline Hip Y-Axis & 41042.2 & $(26018.3)$ \\
Wrist VM & 262098.0 & $(115496.7)$ \\
Hip VM & 82685.8 & $(434339.0)$ \\
$V M:$ & &
\end{tabular}

$V M$ : Vector Magnitude

The mean VM cpm and the total VM counts from the wrist were significantly different from the mean cpm and the total counts recorded from the hip worn accelerometer $(p<0.5)$ and it can be seen that the volume of data is higher when the accelerometer is positioned at the wrist, which is not unexpected given that upper limb movements are likely to increase acceleration output. Despite the significant difference in the VM total count output between the hip and wrist accelerometers these were significantly correlated $r=0.83,(p<0.01)$ and the mean VM cpm from the wrist and hip were also correlated $r=0.81,(p<0.01)$. However, as Figure 1 (i) and (ii) illustrate there was a large differences between the wrist and hip total count data and between the cpm data from the hip and the wrist with wide limits of agreement (mean (LOA), total VM counts: 179412.1 (23042.1,335782.1); cpm: 4976.4 (1809.4,8143.4)). It can also be seen in these plots that the discrepancy between sites increases as the physical activity level increases. 

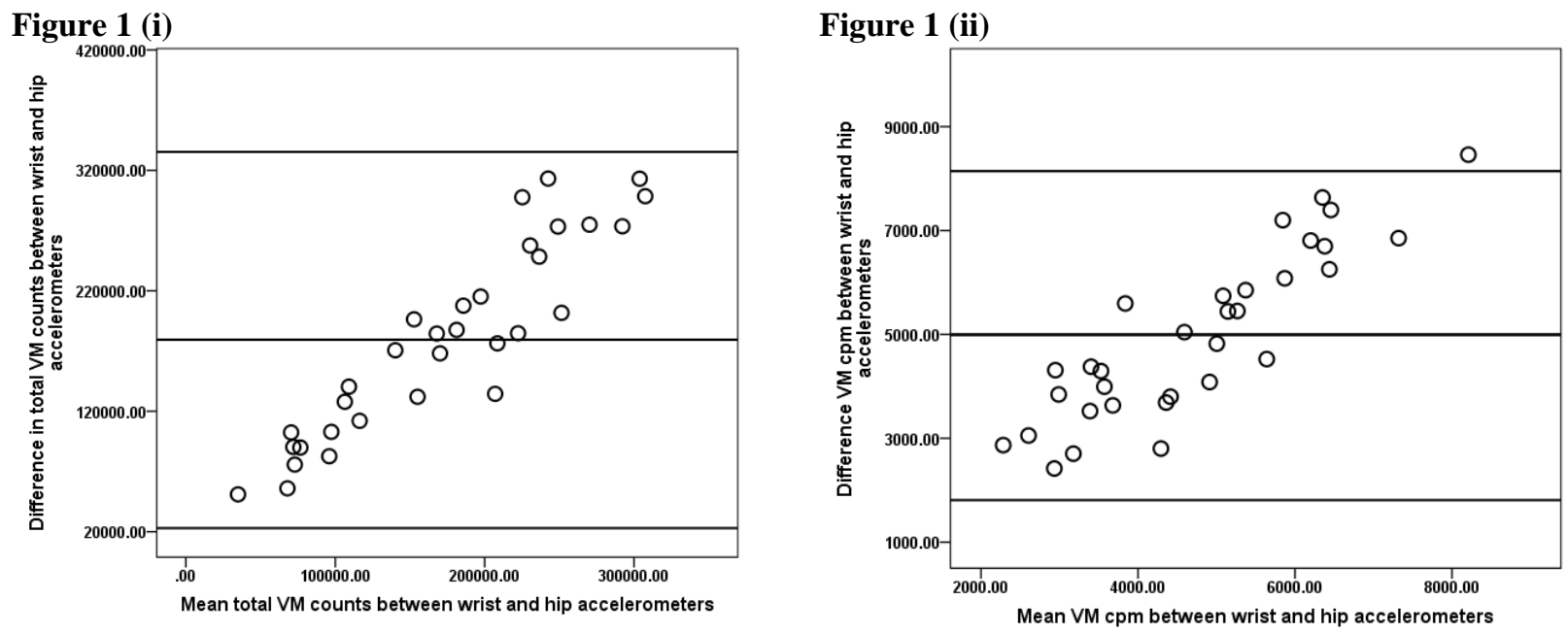

Figure 1: (i) Bland and Altman plot of total vector magnitude (VM) counts between wrist and hip worn accelerometers (ii) Bland and Altman plot of vector magnitude (VM) counts per minute (cpm) between wrist and hip worn accelerometers

Following the application of cut-points, the data on time spent in different intensities and in sedentary behaviour was calculated. The findings are presented in Table 3.

Table 3. Mean minutes (SD) of time spent in different intensities, comparing placement location, cut-points and triaxal and uniaxial output from accelerometers against CARS direct observation scale.

\begin{tabular}{lccc}
\hline & \multicolumn{3}{c}{ Mean minutes (SD) } \\
\hline \multirow{2}{*}{ Intensity } & \multirow{2}{*}{ CARS } & $\begin{array}{c}\text { Johansson } \text { et al (2013) } \\
\text { Wrist, triaxial }\end{array}$ & $\begin{array}{c}\text { Evenson } \text { et al (2008) } \\
\text { Hip, unaxial }\end{array}$ \\
\hline Sedentary & $6.2(5.8)$ & $7.2(3.9)$ & $12.2(7.2)$ \\
Light & $27.3(10.6)$ & $16.9(6.6)$ & $17.2(6.6)$ \\
MVPA & $2.2(2.2)$ & $11.5(6.3)$ & $6.1(4.5)$ \\
TPA & $29.5(10.4)$ & $28.4(9.8)$ & $23.3(9.8)$ \\
\hline
\end{tabular}

CARS, Children's Activity Rating Scale. MVPA, moderate to vigorous activity; TPA, total physical activity.

It can be seen that there is variability in accelerometry estimates of time spent in the different intensities and in sedentary behaviour. While the wrist estimates of sedentary behaviour were similar to the CARS estimate, the hip position resulted in a greater estimate of time spent in sedentary behaviour.

The accelerometer estimates were greater for time spent in high or MVPA than was observed using the CARS. Data from the wrist worn accelerometer using the Johansson et al (2013) cut-points for MVPA is higher than the estimate from the hip worn accelerometer using the Evenson et al (2008) cut-point. Figure 2 illustrates the spread of the data plotting accelerometer estimates from the hip and wrist against the CARS for MVPA and for TPA. 
Figure 2 (i)

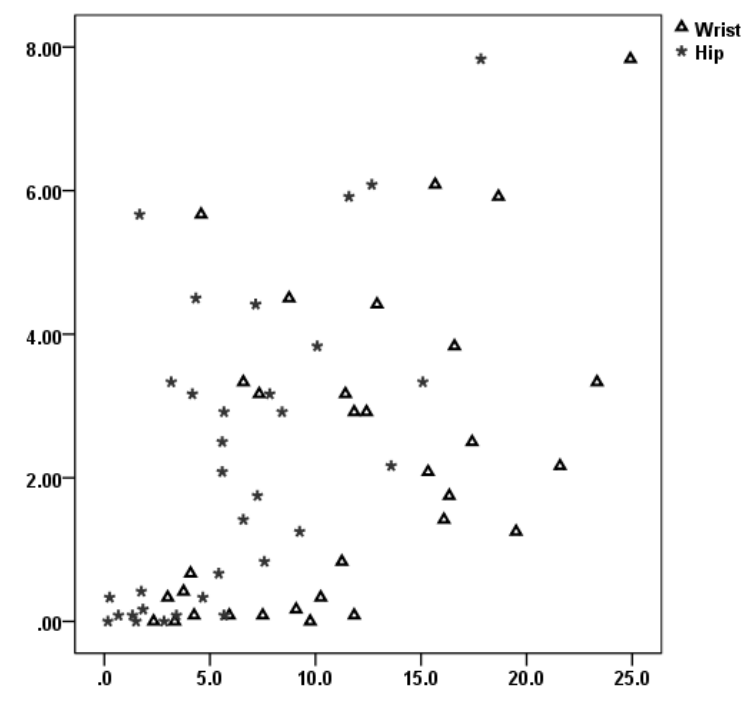

Figure 2 (ii)

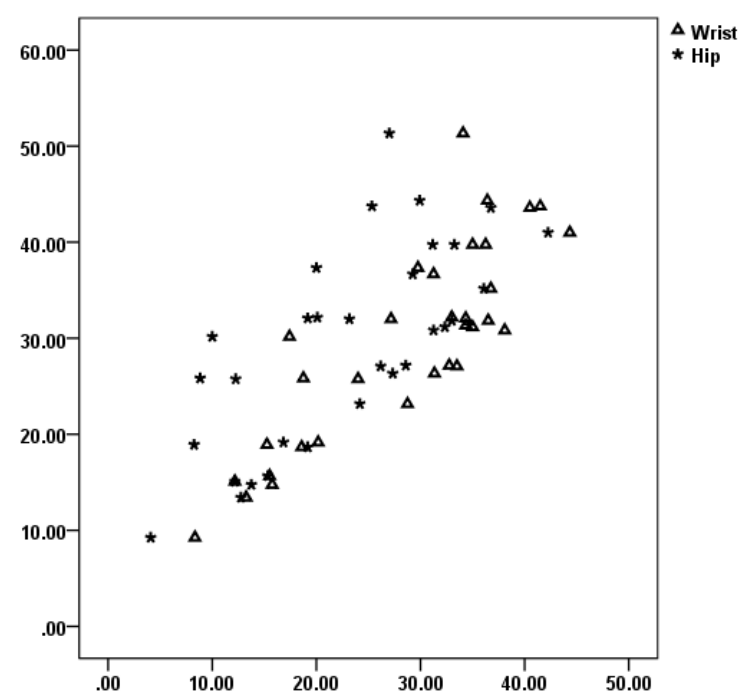

Figure 2: (i) CARS for time spent in MVPA (mins) plotted on y axis, against accelerometer estimates for wrist and hip ( $x$ axis). (ii) CARS for time spent in total physical activity (TPA) (mins) plotted on y axis against accelerometer estimate for hip and wrist ( $x$ axis).

The majority of the time spent in TPA was categorized as the child engaging in 'light' intensity physical activity. To further examine activities within TPA, the time spent in standing and in locomotion was determined. Standing was all activity coded as CARS level 2 and locomotion was all translocation activities (categorized as $\geq 3$, including slow and fast walking). The time spent in these activities was then compared against the accelerometry estimates for the wrist and hip using the Johansson et al (2013) Evenson et al (2008) cut-points respectively and are presented in table 4.

Table 4 Time spent in standing and in locomotion, compared with accelerometry estimates from the wrist and hip worn accelerometers.

\begin{tabular}{lc}
\hline Activity & Mean minutes (SD) \\
\hline Standing activities (CARS 2) (Puhl et al 1990) & $20.0(9.3)$ \\
Wrist 'low' intensity PA (Johansson et al 2013) & $16.9(6.6)$ \\
Hip 'light' intensity PA (Evenson et al 2008) & $17.2(6.6)$ \\
Locomotion activities (CARS $\geq 3$ ), (Puhl et al 1990) & $9.5(5.5)$ \\
Wrist 'high' intensity PA (Johansson et al 2013) & $11.5(6.3)$ \\
Hip 'MVPA', (Evenson et al 2008) & $6.1(4.5)$ \\
\hline
\end{tabular}

CARS, Children's Activity Rating Scale. MVPA, moderate to vigorous activity; $P A$, physical activity. 
To explore the agreement between the estimates and the CARS criterion method, mean differences and 95\% limits of agreement were calculated between the CARS and the accelerometer estimates and are presented in table 5.

Table 5: Mean difference and 95\% limits of agreement (LOA) between wrist and hip estimates and CARS for Total Physical Activity and for MVPA.

\begin{tabular}{lcc}
\hline & \multicolumn{2}{c}{ Time (mins) } \\
\cline { 2 - 3 } & $\begin{array}{c}\text { Mean } \\
\text { difference }\end{array}$ & 95\% LOA \\
\hline TPA CARS - wrist estimate (Johansson et al 2013) & 1.1 & $-9.9,12.1$ \\
TPA CARS - hip estimate (Evenson et al 2008) & 6.3 & $-8.8,21.4$ \\
MVPA CARS - wrist estimate (Johansson et al 2013) & -9.3 & $-20.0,1.5$ \\
MVPA CARS - hip estimate (Evenson et al 2008) & -3.8 & $-10.7,3.1$ \\
Locomotion CARS - wrist estimate 'high' (Johansson et al 2013) & -2.0 & $-9.4,5.4$ \\
Locomotion CARS - hip estimate 'MVPA' (Evenson et al 2008) & 3.4 & $-3.1,10.0$ \\
Standing CARS - wrist estimate 'low' intensity (Johansson et al 2013) & 3.1 & $-14.3,19.9$ \\
Standing CARS - hip estimate light intensity (Evenson et al 2008) & 2.8 & $-10.3,16.4$ \\
\hline CARS, Children's Activity Rating Scale. TPA, total physical activity; MVPA, moderate to vigorous activity, LOA, limits of \\
agreement.
\end{tabular}

Using a repeated measures ANOVA revealed a significant difference between the cut-point estimates $(p<0.01)$ and post-hoc analysis using a paired $t$-test revealed significant differences between each of the pairs of cut-point estimates $(p<0.01)$. While a strong and significant correlation was found between the Evenson et al(2008) estimate from the hip worn accelerometry output and the Johansson et al (2013) estimate from the wrist worn accelerometry output for TPA $\quad(r=0.9, p<0.01)$, the mean difference and and limits of agreement were wide. This could suggest a systematic bias with the wrist accelerometer recording higher estimates physical activity in comparison to the hip worn accelerometer when the Johansson et al (2013) and the Evenson et al (2008) cut-points are used to process accelerometry count data collected from the wrist and hip respectively.

Good agreement was found between the CARS estimate of TPA (29.5 (10.4) mins) and the wrist estimate, using the Johansson et al (2013) cut points (28.4 (9.8) mins). In addition, there was good agreement between the CARS estimate for locomotion activities (9.5 (5.5) mins) and 'high' intensity PA as estimated by the wrist worn accelerometer using the Johansson et al (2013) cut-points (11.5 (6.3) mins). This findings are presented in Figure 3 (i) and (ii). 
Figure 3(i)

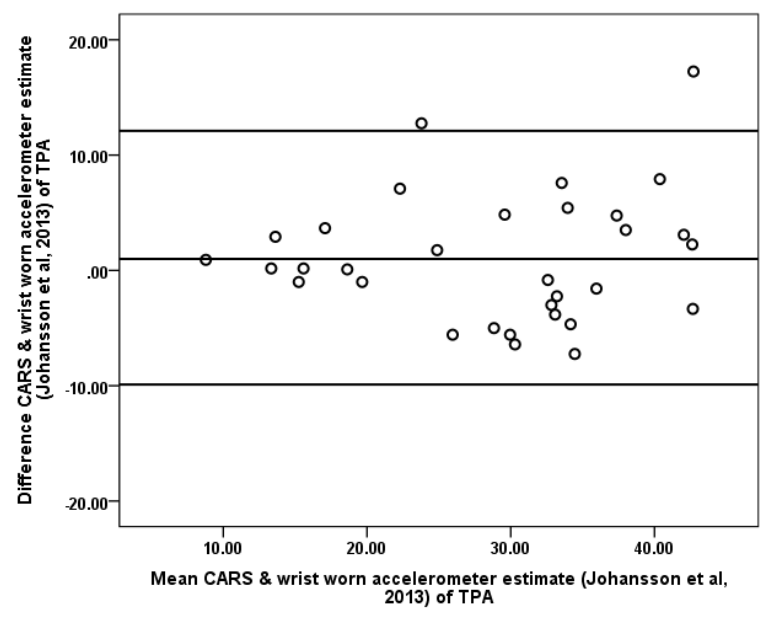

Figure 3(ii)

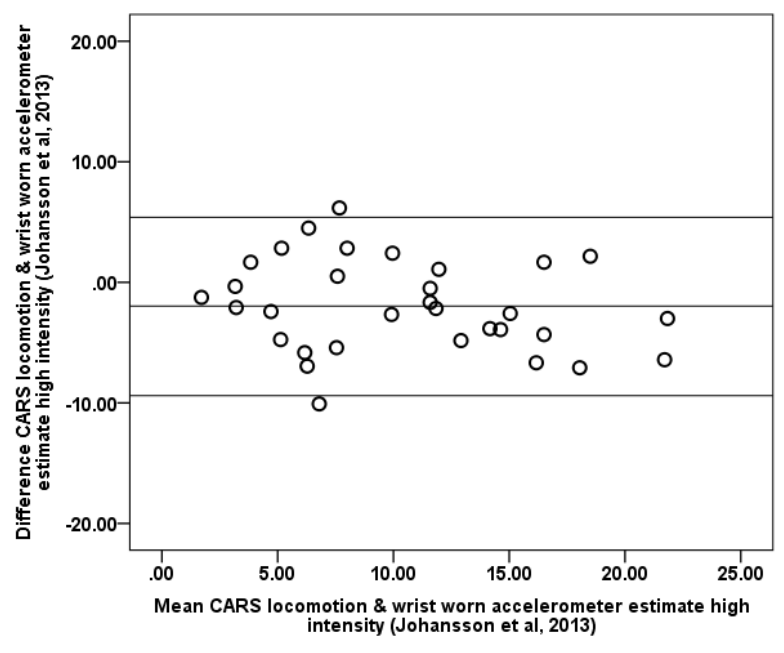

Figure 3:

(i) comparison between the CARS (Puhl et al 1990) wrist worn accelerometer estimate using the Johansson et al (2013) cut-point for total physical activity

(ii) comparison between the CARS (Puhl et al 1990) locomotion and the wrist worn accelerometer estimate for high intensity physical activity using the Johansson et al (2013) cut-points.

\section{Discussion}

The purpose of this study was to investigate agreement between Actigraph estimates of physical activity intensity and sedentary behaviour from wrist and hip-worn Actigraph accelerometers in preschool children during free-play. In addtion the study set out to investigate the validity of the Johansson et al (2013) cut-points for wrist worn Actigraph accelerometers in preschool children aged 3-5 years. The study found that the total VM count output and the mean VM cpm data collected from the wrist and the hip were significantly correlated $(p<0.01)$, however a large and systematic difference, with wide limits of agreement were found between the hip and wrist data. Similar findings have been reported in studies which have involved older children and using other models of accelerometers (Routen et al 2012, Rowlands et al 2014, Ekblom et al 2012). There was good agreement between the CARS and the wrist worn Actigraph estimate of TPA when the Johansson et al (2013) cut-points were applied . There was reasonable group level agreement with the estimates from the hip when Evenson et al (2008) cut-points were used. Notably the agreement at the wrist using the Johannsson et al (2013) and the CARS was better than the hip position estimates when the Evenson et al (2008) cut-points were applied. 
One of the outstanding methodological questions regarding accelerometry data processing and interpretation is how to transform the accelerometry count output into a biologically meaningful format (Cliff et al 2009). While one approach is to apply count thresholds, there is, however, a lack of consensus over which cut-points should be used (Kim et al 2012) and numerous cut-points for Actigraph accelerometers have been developed for children, including pre-school children. The application of different cut-points makes comparison between studies problematic, leading to conflicting conclusions about levels of sedentary behaviour, MVPA and total physical activity (TPA) and without agreement on cut-points it is difficult to ascertain whether children are meeting physical activity guidelines or not (Kim et al 2012, Beets et al 2011).

It is recognised that cut-points are specific to the sample from which they have been calibrated and these also depend on the protocol from which they have been developed (Mackintosh et al 2012). One example is the variability in processing and interpretation of the CARS in calibration studies of accelerometers with preschool children and in particular differences in the classification of sedentary behaviour. In the original CARS scoring system, there is no distinction made between sitting and standing and both these stationary activities, which are level 1 and 2 activities, are classified as being 'sedentary' (Puhl et al 1990). However, more recent consensus is that 'true' sedentary behaviour should be defined as sitting or reclining activities where the resting metabolic rate is typically $\leq 1.5$ METS (Sedentary Behaviour Research Network 2012). Hence a modified CARS approach has been adopted in studies to make the distinction between sitting (level 1) and standing (level 2) activities, where standing is classified as a 'light' intensity and thus part of TPA (Johansson et al 2013). Researchers need to be aware that there may be variation in the interpretation of sedentary behaviour used in calibration studies utilising the CARS and this may contribute to differences in cut-point thresholds used to estimate sedentary time and time spent in TPA. Ideally, to compare physical activity estimates using accelerometers worn at the wrist and hip, it would be best to use cut-points developed from the same study (e.g. using the same sample and protocol). At present, this is not available and thus the cut-points applied in the current study are based on different populations and calibration methods. Despite this, the current study adds to our understanding of how estimates of physical activity compare from studies which have used hip and wrist worn accelerometers.

There was no clear agreement seen between time spent in MVPA with the CARS and the wrist worn accelerometry estimate for high intensity PA using the Johansson et al (2013) cut-points (mean difference 
(LOA): -9.3, (-20.0, 1.5)). Similarly a difference was found between the time spent in MVPA using the CARS and the hip worn accelerometry estimate with the Evenson et al (2008) cut-points (mean difference (LOA): -3.8 (-10.7, 3.1)). However, there was better agreement was found for time spent in translocation or locomotion (including both slow and fast walking) using the CARS and the Johansson et al (2013) estimate for high intensity physical activity (mean difference (LOA): -2.0 (-9.4,5.4)). While the interpretation of what constitutes MVPA or high intensity PA needs careful consideration, the wrist worn accelerometer demonstrated good agreement with locomotion activities in this preschool population when the Johansson et al (2013) cut-points were applied.

To allow for standardisation in the assessment of physical activity between studies Bassett et al (2015) has argued for the reporting of total volume of activity counts per day (TAC/d). This would provide a more direct measure of physical activity and avoid the issues of applying different cut-point or algorithm to accelerometry data which can result in large apparent difference in physical activity outcomes (Beets et al 2011, Bornstein et al 2011). The findings of this study suggest that there are wide discrepancies between the volume of count data collected from the wrist and the hip. Given the differences of 184978.1(2114.4,367841.8) counts from 1 hour of data collection, it would be substantially higher over a longer period of data collection. However, these differences are not unexpected and indeed the higher wrist worn may help to capture more accurately total physical activity of preschool children. However, how this count data collected from the wrist relates to uniaxial Actigraph data already collected from the hip warrants further investigation, if trends or patterns in physical activity behaviour is to be explored over time.

There are a number of limitations in this study. The first is that this study was limited to 1 hour of observation of physical activity behaviour of children in a nursing setting. As such further investigation to compare wrist and hip accelerometry over a longer period of time, in day to day living, is important in gaining greater understanding of the relationship between monitor output. Secondly the CARS as a criterion measure has been questioned (Adolph et al 2012, Oliver et al 2007). Adolph et al. (2012) argues that while direct observation is critical for identification of type of activity; this method lacks precision in quantifying intensity levels and thus energy expenditure. Moreover, Oliver et al. (2007) argues that direct observation should be considered as a subjective method, as it relies on the observer to observe, interpret and code children's physical activity behaviour. Despite this, given the problems with interpreting energy expenditure in young children, observational methods offer a behavioural approach to 
calibration as an alternative. In addition, this approach is argued to be valuable in studies of young children, particularly if there is an interest in 'type' or 'patterns' of physical activity (Freedson et al 2005).

Another limitation of the study is that the data were collected in 1-s epochs and then re-integrated into 5-s epochs, in addition cut-points values which were validated for 60-s epochs (Puyau et al 2002) or for 15-s epochs (Evenson et al 2008) were divided into 5-s epochs and whether this creates errors in the data processing has been raised as a concern (Kim et al 2013).

With the availability of raw accelerometry data it has been argued that rather than reporting the 'count' output which, varies between the models of accelerometer and trying to convert this to a biologically meaningful format, future accelerometers should provide data in standardised units such as gravitational constant $(\mathrm{G}, \mathrm{m} \cdot \mathrm{s}-2)$ or time-integrated units $(\mathrm{m} \cdot \mathrm{s}-1)$. This would allow for greater ease of comparison between accelerometry output between models. The use of raw accelerometry data instead of activity counts had been proposed for incorporation within a consensus statement at the 2009 'Objective Measurement of Physical Activity: Best Practice and Future Directions' conference (John and Freedson 2012). One possible concern with using raw acceleration data is how to manage the raw acceleration data and convert this into a meaningful format for the 'end user' be it researcher or policy-maker. Advances have, however, been made in accelerometer data processing with the development of more sophisticated approaches to data modelling analysis (Bonomi et al 2009, Pober et al 2006, Staudenmayer et al 2009, Zhang et al 2003). This area warrants further investigation in studies of pre-school children to determine if this will offer an accurate means of classifying physical activity behaviour.

Although it is agreed that accelerometer placement has an effect on the measurement of bodily acceleration, there is still some debate over the ideal location of the sensor for particular applications (Cleland et al 2013). A strength this study, is that it is one of the few studies to explore the relationship between output from hip and wrist worn Actigraph accelerometers and to date the first exploring this in the preschool population. Greater understanding of this relationship is important for longitudinal comparison of physical behaviour over time. Finally, valid cut-points for wrist worn Actigraph accelerometers had not been previously established in this population.

In conclusion, the findings of this study suggest that while the output from wrist and hip Actigraph accelerometers were significantly correlated but that there was large bias between the VM count data and the VM cpm data collected from the two placement sites. There was systematic bias with differences accentuated with the wrist higher total VM count and VM cpm data. There is reasonable agreement 
between the wrist and the hip estimates of TPA when Johansson et al (2013) is applied to the VM data collected at the wrist and the Evenson et al (2008) cut-points are applied to the uniaxial data at the hip. However, this warrants further investigation over a longer duration.

Finally, good agreement was found between the Johansson et al (2013) and a modified version of the CARS, where only sitting activities are classified as sedentary and standing activities were classified as part of 'TPA'. This suggests good validity for Johansson et al (2013) cut-points for wrist worn Actigraph accelerometers in preschool children aged 3-5 years at a group level.

\section{References}

Adolph A L, Puyau M R, Vohra F A, Nicklas T A, Zakeri I F and Butte N F 2012 Validation of uniaxial and triaxial accelerometers for the assessment of physical activity in preschool children J. Phys. Act. Health 9 944-53

Alhassan S, Sirard J R and Robinson T N 2007 The effects of increasing outdoor play time on physical activity in Latino preschool children Int J Pediatr Obes 2 153-8

Australian Government, Department of Health and Ageing 2010 Get up and grow: healthy eating and physical activity for early childhood. Directors/coordinators book http://www.health.gov.au/internet/main/publishing.nsf/Content/

Bassett D R, Troiano R P, Mcclain J J and Wolff D L 2015 Accelerometer-based Physical Activity: Total Volume per Day and Standardized Measures Med Sci Sports Exs 47 833-8

Basterfield L, Adamson A J, Frary J K, Parkinson K N, Pearce M S, Reilly J J and Gateshead Millennium Study Core Team 2011 Longitudinal study of physical activity and sedentary behavior in children Pediatrics 127 e24-30

Beets M W, Bornstein D, Dowda M and Pate R R 2011 Compliance with national guidelines for physical activity in U.S. preschoolers: measurement and interpretation Pediatrics 127 658-64

Biddle S J, Pearson N, Ross G M and Braithwaite R 2010 Tracking of sedentary behaviours of young people: a systematic review Prev. Med. 51 345-51

Bonomi A G, Goris A H, Yin B and Westerterp K R 2009 Detection of type, duration, and intensity of physical activity using an accelerometer Med. Sci. Sports Exerc. 41 1770-7

Bornstein D B, Beets M W, Byun W and McIver K 2011 Accelerometer-derived physical activity levels of preschoolers: a meta-analysis J. Sci. Med. Sport 14 504-11

Canadian Society of Exercise Physiology 2012 Canadian physical activity guidelines: for the early years - 0-4 years http://www.csep.ca/CMFiles/Guidelines/CSEP-InfoSheets-early-years-ENG.pdf

Cleland I, Kikhia B, Nugent C, Boytsov A, Hallberg J, Synnes K, McClean S and Finlay D 2013 Optimal Placement of Accelerometers for the Detection of Everyday Activities Sensors 13 9183-200 
Cliff D P, Reilly J J and Okely A D 2009 Methodological considerations in using accelerometers to assess habitual physical activity in children aged 0-5 years J. Sci. Med. Sport 12 557-67

Cole T J 2002 A chart to link child centiles of body mass index, weight and height Eur. J. Clin. Nutr. 1194-9

Cox R, Skouteris H, Rutherford L, Fuller-Tyszkiewicz M, Dell'Aquila D and Hardy L L 2012 Television viewing, television content, food intake, physical activity and body mass index: a cross-sectional study of preschool children aged 2-6 years Health Promot J Austr 23 58-62

Dale D, Corbin C B and Dale K S 2000 Restricting opportunities to be active during school time: do children compensate by increasing physical activity levels after school? Res. Q. Exerc. Sport 71 240-8

De Decker E, De Craemer M, Santos-Lozano A, Van Cauwenberghe E, De Bourdeaudhij I and Cardon G 2013 Validity of the activPALTM and the Actigraph monitors in preschoolers Med. Sci. Sports Exerc. [Epub ahead of print]

de Vries S I, Bakker I, Hopman-Rock M, Hirasing R A and van M W 2006 Clinimetric review of motion sensors in children and adolescents J. Clin. Epidemiol. 59 670-80

de Vries S I, Van Hirtum H W, Bakker I, Hopman-Rock M, Hirasing R A and van M W 2009 Validity and reproducibility of motion sensors in youth: a systematic update Med. Sci. Sports Exerc. 41 818-27

Department of Health, Physical Activity, Health Improvement and Protection 2011 Start Active, Stay Active. A report on physical activity for health from the four home countries' Chief Medical Officers http://www.dh.gov.uk/en/Publicationsandstatistics/Publications/PublicationsPolicyAndGuidance/DH_128209

Dietz W H 2001 The obesity epidemic in young children. Reduce television viewing and promote playing $B M J 322$ 313-4

Djafarian K, Speakman J R, Stewart J and Jackson D M 2013 Comparison of activity levels measured by a wrist worn accelerometer and direct observation in young children OJPed $\mathbf{3} 422$

Ekblom O, Nyberg G, Bak E E, Ekelund U and Marcus C 2012 Validity and Comparability of a Wrist-Worn Accelerometer in Children J. Phys. Act. Health $9389-93$

Evenson K R, Catellier D J, Gill K, Ondrak K S and McMurray R G 2008 Calibration of two objective measures of physical activity for children J. Sports Sci. 26 1557-65

Freedson P, Pober D and Janz K F 2005 Calibration of accelerometer output for children Med. Sci. Sports Exerc. 37 S523-30

Freedson P S and Miller K 2000 Objective monitoring of physical activity using motion sensors and heart rate Res. Q. Exerc. Sport 71 S21-9

Goldfield G S, Harvey A, Grattan K and Adamo K B 2012 Physical activity promotion in the preschool years: a critical period to intervene Int. J. Environ Res Public Health 9 1326-42

Heelan K A and Eisenmann J C 2006 Physical activity, media time, and body composition in young children $J$. Phys. Act. Health 3 200-9

Hildebrand M, Van Hees V T, Hansen B H and Ekelund U 2014 Age Group Comparability of Raw Accelerometer Output from Wrist- and Hip-Worn Monitors Med. Sci. Sports Exerc. 46 1816-24 
Hinkley T, Salmon J, Okely A, D., Crawford D and Hesketh K 2012 Preschoolers' physical activity, screen time, and compliance with recommendations Med. Sci. Sports Exerc. 44 458-65

Hislop J F, Bulley C, Mercer T H and Reilly J J 2012 Comparison of epoch and uniaxial versus triaxial accelerometers in the measurement of physical activity in preschool children: a validation study Pediatr. Exerc. Sci. 24 450-60

Jackson D M, Reilly J J, Kelly L A, Montgomery C, Grant S and Paton J Y 2003 Objectively measured physical activity in a representative sample of 3- to 4-year-old children Obes. Res. 11 420-5

Janz K F, Burns T L, Levy S M, Torner J C, Willing M C, Beck T J, Gilmore J M and Marshall T A 2004 Everyday activity predicts bone geometry in children: the Iowa bone development study Med. Sci. Sports Exerc. 36 1124-31

Janz K F, Gilmore J M, Burns T L, Levy S M, Torner J C, Willing M C and Marshall T A 2006 Physical activity augments bone mineral accrual in young children: The Iowa Bone Development study J. Pediatr. 148 793-9

Janz K F, Levy S M, Burns T L, Torner J C, Willing M C and Warren J J 2002 Fatness, physical activity and television viewing in children during the adiposity rebound period: the Iowa bone development study Prev. Med. 35 536-71

Johansson E, Ekelund U, Nero H, Marcus C and Hagströmer M 2013 Calibration and cross-validation of a wrist-worn Actigraph in young preschoolers Pediatr Obes 10 1-6

John D and Freedson P 2012 Actigraph and Actical physical activity monitors: a peek under the hood Med. Sci. Sports Exerc. 44 S86-9

Kim Y, Beets M W, Pate R R and Blair S N 2013 The effect of reintegrating Actigraph accelerometer counts in preschool children: comparison using different epoch lengths J Sci Med Sports 16 129-34

Kim Y, Beets M W and Welk G J 2012 Everything you wanted to know about selecting the "right" Actigraph accelerometer cut-point for youth, but....A systematic review PLoS one 15 311-21

Mackintosh, K A, Fairclough, S J, Stratton, G and Ridgers, N D 2012 A calibration protocol for population-specific accelerometer cut-points in children PLoS one 7 e36919

Malina R M 1996 Tracking of physical activity and physical fitness across the lifespan Res. Q. Exerc. Sport 67 S48-57

Martinez-Gomez D, Tucker J, Heelan K A, Welk G J and Eisenmann J C 2009 Associations between sedentary behavior and blood pressure in young children Arch. Pediatr. Adolesc. Med. 163 724-30

Mattocks C, Hines M, Ness A, Leary S, Griffiths A, Tilling K, Blair S N and Riddoch C 2010 Associations between sex-typed behaviour at age $31 / 2$ and levels and patterns of physical activity at age 12: the Avon Longitudinal Study of Parents and Children Arch. Dis. Child. 95 509-12

Metallinos-Katsaras E S, Freedson P, Fulton J E and Sherry B 2007 The association between an objective measure of physical activity and weight status in preschoolers Obesity 15 686-94

National Association for Sport and Physical Education 2009 Active Start: a statement of physical activity guidelines for children from birth to age 5 2nd edn (Reston, VA: American Alliance for Health, Physical Education, Recreation and Dance) 
National Institute for Health and Clinical Excellence 2009 PH 17 Promoting physical activity for children and young people: quick reference guide http://www.nice.org.uk/nicemedia/live/11773/42885/42885.PDF

Obeid J, Nguyen T, Gabel L and Timmons B W 2011 Physical activity in Ontario preschoolers: prevalence and measurement issues Appl Physiol Nutr Metab 36 291-7

Ojiambo R et al 2011 Impact of methodological decisions on accelerometer outcome variables in young children Int. J. Obes. 35 S $98-\mathrm{S} 103$

Okely A D, Trost S G, Steele J R, Cliff D P and Mickle K 2009 Adherence to physical activity and electronic media guidelines in Australian pre-school children J. Paediatr. Child Health 45 5-8

Oliver M, Schofield G M and Kolt G S 2007 Physical activity in preschoolers: understanding prevalence and measurement issues Sports Med. 37 1045-70

Parsons T J, Power C, Logan S and Summerbell C D 1999 Childhood predictors of adult obesity: a systematic review Int. J. Obes. 23 Suppl 8 S1-107

Pate R R, Almeida M J, McIver K L, Pfeiffer K A and Dowda M 2006 Validation and calibration of an accelerometer in preschool children Obesity 14 2000-6

Pate R R, O'Neill J R and Mitchell J 2010 Measurement of physical activity in preschool children Med. Sci. Sports Exerc. 42 508-12

Pfeiffer K A, McIver K L, Dowda M, Almeida M J and Pate R R 2006 Validation and calibration of the Actical accelerometer in preschool children Med. Sci. Sports Exerc. 38 152-7

Pober D M, Staudenmayer J, Raphael C and Freedson P S 2006 Development of novel techniques to classify physical activity mode using accelerometers Med. Sci. Sports Exerc. 38 1626-34

Puhl J, Greaves K, Hoyt M and Baranowski T 1990 Children's Activity Rating Scale (CARS): description and calibration Res. Q. Exerc. Sport 61 26-36

Puyau M R, Adolph A L, Vohra F A and Butte N F 2002 Validation and calibration of physical activity monitors in children Obes. Res. 10 150-7

Rokholm B, Baker J L and Sørensen T I A 2010 The levelling off of the obesity epidemic since the year 1999- a review of evidence and perspectives Obes Rev 11 835-46

Routen A C, Upton D, Edwards M G and Peters D M 2012 Discrepancies in accelerometer measured physical activity in children due to cut-point non-equivalence and placement site J. Sports Sci. 30 1303-10

Rowlands A V, Rennie K, Kozarski R, Stanley R M, Eston R G, Parfitt G C and Olds T S 2014 Children's Physical Activity Assessed with Wrist- and Hip-Worn Accelerometers Med Sci Sports Exs 46 2308-16

Sedentary Behaviour Research Network 2012 Letter to the Editor: standardized use of the terms "sedentary" and "sedentary behaviours" Appl Physiol Nutr Metab 37 540-2

Sherar L B, Griew P, Esliger D W, Cooper A R, Ekelund U, Judge K and Riddoch C 2011 International children's accelerometry database (ICAD): design and methods BMC Public Health 11485 
Sirard J R, Trost S G, Pfeiffer K A, Dowda M and Pate R R 2005 Calibration and evaluation of an objective measure of physical activity in preschool children J Phys Activ Health; J. Phys. Act. Health 2 345-57

Staudenmayer J, Pober D, Crouter S E, Bassett D R and Freedson P 2009 An artificial neural network to estimate physical activity energy expenditure and identify physical activity type from an accelerometer J. Appl. Physiol. 107 1300-7

Taylor R W, Murdoch L, Carter P, Gerrard D F, Williams S M and Taylor B J 2009 Longitudinal study of physical activity and inactivity in preschoolers: the FLAME study Med. Sci. Sports Exerc. 41 96-102

Telford A, Salmon J, Timperio A and Crawford D 2005 Examining physical activity among 5- to 6- and 10- to 12-year-old children: the Children's Leisure Activities Study Pediatr. Exerc. Sci. 17 266-80

Trost S G, Loprinzi P D, Moore R and Pfeiffer K A 2011 Comparison of accelerometer cut points for predicting activity intensity in youth Med. Sci. Sports Exerc. 43 1360-8

Trost S G, Zheng Y L and Wong W K 2014 Machine learning for activity recognition: hip versus wrist data Physiol Meas. 35 2183-9

Zhang K, Werner P, Sun M, Pi-Sunyer F X and Boozer C N 2003 Measurement of human daily physical activity Obes. Res. 11 33-40 